\title{
Memory-built-in quantum teleportation with photonic and atomic qubits
}

\author{
YU-A0 CHEN ${ }^{1,2 *}$, SHUAI CHEN ${ }^{1}$, ZHEN-SHENG YUAN ${ }^{1,2}$, B0 ZHA0 ${ }^{1}$, CHIH-SUNG CHUU ${ }^{1}$, \\ JÖRG SCHMIEDMAYER ${ }^{3}$ AND JIAN-WEI PAN ${ }^{1,2 *}$
}

\author{
${ }^{1}$ Physikalisches Institut, Universität Heidelberg, Philosophenweg 12, D-69120 Heidelberg, Germany \\ ${ }^{2}$ Hefei National Laboratory for Physical Sciences at Microscale and Department of Modern Physics, University of Science and Technology of China, Hefei, Anhui 230026, \\ People's Republic of China \\ ${ }^{3}$ Atominstitut der Österreichischen Universitäten, TU-Wien, A-1020 Vienna, Austria \\ *e-mail: yuao@physi.uni-heidelberg.de; jian-wei.pan@physi.uni-heidelberg.de
}

The combination of quantum teleportation ${ }^{1}$ and quantum memory ${ }^{2-5}$ of photonic qubits is essential for future implementations of large-scale quantum communication ${ }^{6}$ and measurement-based quantum computation ${ }^{7,8}$. Both steps have been achieved separately in many proof-of-principle experiments $^{9-14}$, but the demonstration of memory-built-in teleportation of photonic qubits remains an experimental challenge. Here, we demonstrate teleportation between photonic (flying) and atomic (stationary) qubits. In our experiment, an unknown polarization state of a single photon is teleported over $7 \mathrm{~m}$ onto a remote atomic qubit that also serves as a quantum memory. The teleported state can be stored and successfully read out for up to $8 \mu \mathrm{s}$. Besides being of fundamental interest, teleportation between photonic and atomic qubits with the direct inclusion of a readable quantum memory represents a step towards an efficient and scalable quantum network ${ }^{2-8}$.

Quantum teleportation ${ }^{1}$, a way to transfer the state of a quantum system from one place to another, was first demonstrated between two independent photonic qubits ${ }^{9}$; later developments include demonstration of entanglement swapping ${ }^{10}$, open-destination teleportation ${ }^{11}$ and teleportation between two ionic qubits ${ }^{15,16}$. Teleportation has also been demonstrated for a continuous-variable system, that is, transferring a quantum state from one light beam to another ${ }^{17}$ and, more recently, even from light to matter ${ }^{18}$.

However, the above demonstrations have several drawbacks, especially in long-distance quantum communication. On the one hand, the absence of quantum storage makes the teleportation of light alone non-scalable. On the other hand, in teleportation of ionic qubits, the shared entangled pairs were created locally, which limits the teleportation distance to a few micrometres and is difficult to extend to large distances. In continuous-variable teleportation between light and matter, the experimental fidelity is extremely sensitive to the transmission loss-even in the ideal case, only a maximal attenuation of $10^{-1}$ is tolerable ${ }^{19}$. Moreover, the complicated protocol required for retrieving the teleported state in the matter ${ }^{20}$ is beyond the reach of current technology.

The combination of quantum teleportation and quantum memory of photonic qubits ${ }^{2-5}$ could provide a novel way to overcome these drawbacks. Here, we achieve this appealing combination by experimentally implementing teleportation between discrete photonic (flying) and atomic (stationary) qubits.
In our experiment, we use the polarized photonic qubits as the information carriers and the collective atomic qubits ${ }^{2-5,12}$ (an effective qubit consists of two atomic ensembles, each with $10^{6}$ rubidium-87 atoms) as the quantum memory. In memorybuilt-in teleportation, an unknown polarization state of single photons is teleported onto and stored in a remote atomic qubit via a Bell-state measurement between the photon to be teleported and the photon that is originally entangled with the atomic qubit. The protocol has several distinct features. First, different from an ionic system, its information carrier (flying photonic qubit) is robust against decoherence and can be easily transmitted over large distances. Second, different from a continuous-variable system, its teleportation fidelity is insensitive to photon losses. In practice, an overall transmission attenuation of $10^{-4}$ is tolerable with current technology, as demonstrated in recent experiments ${ }^{21,22}$. Moreover, as the collective state of atomic ensembles is used to encode an atomic qubit, the teleported state can be easily read out in a controllable time for further quantum information applications. In principle, these distinct advantages make our method robust for scalable quantum communication and computation networks $\mathrm{s}^{3-5}$.

A schematic diagram of the set-up of our experiment is shown in Fig. 1. At Bob's site, a pair of effective maximally entangled qubits is created by sending two classical light pulses through two atomic ensembles $\mathrm{U}$ (up) and D (down), which are located $0.6 \mathrm{~m}$ apart in two magneto-optical traps (MOTs) for ${ }^{87} \mathrm{Rb}$. The two ground states $|a\rangle\left(5 S_{1 / 2}, F=2\right)$ and $|b\rangle\left(5 S_{1 / 2}, F=1\right)$ form together with the excited level $|e\rangle\left(5 P_{1 / 2}, F^{\prime}=2\right)$ a $\Lambda$-type system. Initially, each ensemble is prepared in the ground state $|a\rangle$. Shining a weak classical write pulse coupling the transition $|a\rangle \rightarrow|e\rangle$ with a red detuning $\Delta(10 \mathrm{MHz})$ and the Rabi frequency $\Omega_{\mathrm{W}}$ into the ensemble $m(m=\mathrm{U}$ or $\mathrm{D})$ creates a superposition between the antiStokes field $\hat{a}_{\mathrm{AS}}$ and a collective spin state of the atoms ${ }^{2}$,

$$
|\Psi\rangle_{m} \sim\left|0_{\mathrm{AS}} 0_{\mathrm{b}}\right\rangle_{m}+\sqrt{\chi_{m}}\left|1_{\mathrm{AS}} 1_{\mathrm{b}}\right\rangle_{m}+O\left(\chi_{m}\right)
$$

where $\chi_{m} \ll 1$ is the excitation probability of one spin flip in ensemble $m$, and $\left|i_{\mathrm{AS}} i_{\mathrm{b}}\right\rangle$ denotes the $i$-fold excitation of the antiStokes field and the collective spin. We adjust $\chi_{U}=\chi_{D}$, select orthogonal polarization of the two anti-Stokes fields and combine them on a polarized beam splitter $\left(\mathrm{PBS}_{1}\right)$, as illustrated in Fig. 1. Neglecting the vacuum state and high-order excitations, the state 


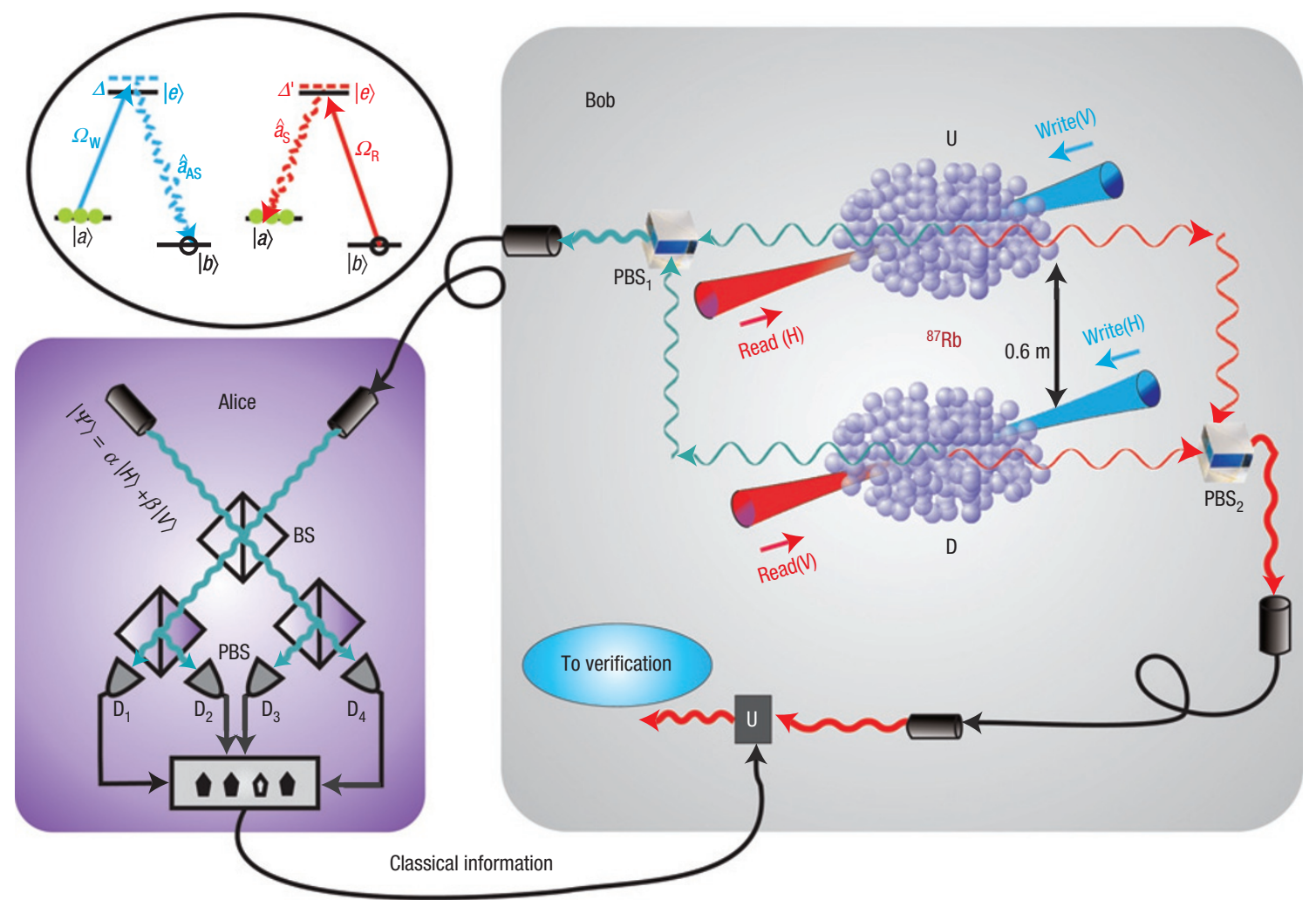

Figure 1 Experimental set-up for teleportation between photonic and atomic qubits. The top-left diagram shows the structure and the initial populations of atomic levels for the two ensembles. At Bob's site, the anti-Stokes fields emitted from $U$ and $D$ are collected and combined at $\mathrm{PBS}_{1}$, selecting perpendicular polarizations. Then the photon travels $7 \mathrm{~m}$ through the fibres to Alice's site to overlap with the initial unknown photon on a beam splitter (BS) to carry out the BSM. The results of the BSM are sent to Bob through a classical channel. Bob then carries out the verification of the teleported state in the $\mathrm{U}$ and $\mathrm{D}$ ensembles by converting the atomic excitation to a photonic state. If the state $\left|\Psi^{+}\right\rangle$is registered, Bob directly carries out a polarization analysis on the converted photon to measure the teleportation fidelity. On the other hand, if the state $\left|\Psi^{-}\right\rangle$is detected, the converted photon is sent through a half-wave plate via the first-order diffraction of an AOM (not shown). The half-wave plate is set at $0^{\circ}$ serving as the unitary transformation of $\hat{\sigma}_{z}$. Then the photon is sent through the polarization analyser to obtain the teleportation fidelity.

of the photonic and atomic qubits can be described as an effectively entangled state

$$
|\Psi\rangle=\frac{1}{\sqrt{2}}(|H\rangle|\tilde{V}\rangle+|V\rangle|\tilde{H}\rangle),
$$

where $|H\rangle(|V\rangle)$ denotes horizontal (vertical) polarizations of single photons and $|\tilde{H}\rangle=\left|0_{\mathrm{b}}\right\rangle_{\mathrm{U}}\left|1_{\mathrm{b}}\right\rangle_{\mathrm{D}}\left(|\tilde{V}\rangle=\left|1_{\mathrm{b}}\right\rangle_{\mathrm{U}}\left|0_{\mathrm{b}}\right\rangle_{\mathrm{D}}\right)$ denotes one spin excitation in ensemble $\mathrm{D}(\mathrm{U})$. Note that the anti-Stokes photon has a coherence time of $25 \mathrm{~ns}$ (ref. 23) and can thus overlap with the photon to be teleported very easily.

After the effectively entangled state equation (1) is prepared, the anti-Stokes photon is sent to Alice over a 7-m-long fibre. Suppose that at Alice's site, the photon to be teleported is in an unknown polarization state $|\phi\rangle=\alpha|H\rangle+\beta|V\rangle$. In terms of four Bell states, $\left|\Psi^{ \pm}\right\rangle=(1 / \sqrt{2})(|H V\rangle \pm|V H\rangle)$ and $\left|\Phi^{ \pm}\right\rangle=(1 / \sqrt{2})(|H H\rangle \pm|V V\rangle)$, the combined state of the three qubits can be rewritten as

$$
\begin{aligned}
|\phi\rangle|\Psi\rangle= & \frac{1}{2}\left(\left|\Phi^{+}\right\rangle \hat{\sigma}_{x}|\tilde{\phi}\rangle+\left|\Phi^{-}\right\rangle\left(-i \hat{\sigma}_{y}|\tilde{\phi}\rangle\right)\right. \\
& \left.+\left|\Psi^{+}\right\rangle|\tilde{\phi}\rangle+\left|\Psi^{-}\right\rangle \hat{\sigma}_{z}|\tilde{\phi}\rangle\right),
\end{aligned}
$$

where $\hat{\sigma}_{x}, \hat{\sigma}_{y}$ and $\hat{\sigma}_{z}$ are the well-known Pauli operators, and $|\tilde{\phi}\rangle=\alpha|\tilde{H}\rangle+\beta|\tilde{V}\rangle$. This shows that a joint Bell-state measurement (BSM) on the two photons at Alice's side projects the state of the atomic qubit at Bob's side into one of the four corresponding states in equation (2). After the BSM, the initial state of the photonic qubit is thus transferred to and stored in the atomic qubit. In standard teleportation, depending on the BSM results, Bob can then carry out a unitary transformation, independent of $|\phi\rangle$, on the atomic qubit to convert its state into the initial state of the photonic qubit.

To achieve the required BSM, the photon from the entangled state (1) and the photon to be teleported are superposed on a 50:50 beam splitter (Fig. 1). The beam splitter together with the subsequent coincidence measurements is capable of identifying two of the four Bell states ${ }^{24},\left|\Psi^{+}\right\rangle$and $\left|\Psi^{-}\right\rangle$in our experiment. Note that to demonstrate the working principle of teleportation, it is sufficient to identify only one of the four Bell-states, for example, through identification of $\left|\Psi^{+}\right\rangle$and verification of $|\tilde{\phi}\rangle$ (refs 9-11).

To verify the success of teleportation, we convert the atomic excitation back to optical excitation in a controllable time by shining in two simultaneous read pulses, coupling the transition $|b\rangle \rightarrow|e\rangle$ with a blue detuning $\Delta^{\prime}(6 \mathrm{MHz})$ and the Rabi frequency $\Omega_{\mathrm{R}}$. The polarizations of the two read pulses are selected to be perpendicular with respect to the corresponding write pulses. The retrieved Stokes fields are then combined at $\mathrm{PBS}_{2}$. Hence, the atomic qubit is converted back to a single-photon polarization qubit. Instead of carrying out a direct measurement on the atomic qubit, via a polarization measurement on the converted single-photon state, we can thus obtain the experimental teleportation fidelity. 


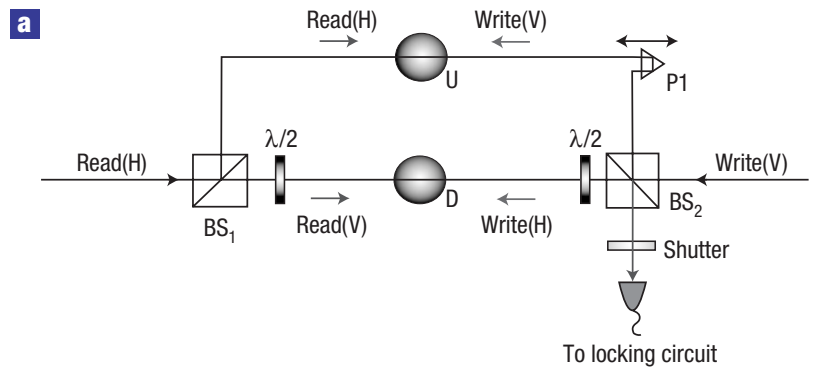

b

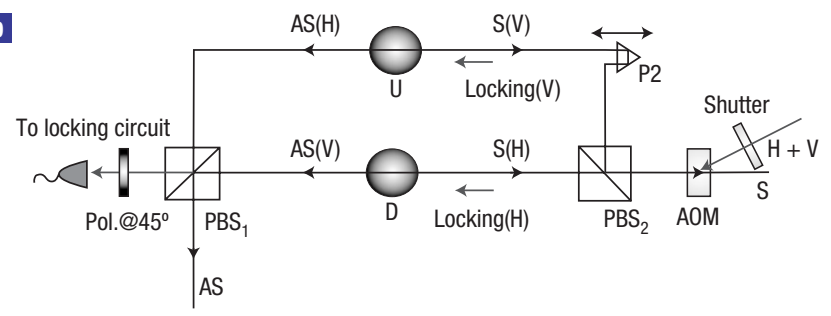

Figure 2 Schematic diagram of the phase-locking set-up. a,b, Two Mach-Zehnder interferometers are used to actively stabilize the phases between the arms of the write and read paths (a) and between the arms of the anti-Stokes and Stokes paths (b), respectively. $\mathrm{H}$ (V) denotes the horizontal (vertical) polarization. A polarizer (Pol.) is set at $45^{\circ}$ to erase the polarization information. The $\lambda / 2$ plates are also set at $45^{\circ}$ to rotate the horizontal polarization to vertical. $\mathrm{AS}(\mathrm{S})$ denotes the anti-Stokes (Stokes) photon.

If teleportation occurs, conditional on detecting a $\left|\Psi^{+}\right\rangle$state at Alice's side, the state of the atomic qubit at Bob's side will be left in the state $|\tilde{\phi}\rangle$ (equation (2)). Following the read-out protocol, the collective atomic state $|\tilde{\phi}\rangle$ will be converted into the initial polarization state $|\phi\rangle$. On the other hand, if a $\left|\Psi^{-}\right\rangle$state is detected, the state of the atomic qubit will then be left in the state $\hat{\sigma}_{z}|\tilde{\phi}\rangle$, which after conversion is equivalent to the initial state except for a unitary transformation $\hat{\sigma}_{z}$. Consequently, applying $\hat{\sigma}_{z}$ on the converted single-photon polarization state, we will again obtain the same initial state $|\phi\rangle$. It is worth noting that the ease of both transferring atomic excitation to optical excitation and exploiting linear optical elements to carry out precise unitary transformation on single-photon states is a distinct advantage of our method.

Before carrying out the teleportation, it is necessary to verify the entanglement. To do so, we map the atomic excitation back into a photon by sending two classical read pulses through the two ensembles. The retrieved Stokes fields with perpendicular polarizations are combined on $\mathrm{PBS}_{2}$ (Fig. 1). And the superposition state of the anti-Stokes and Stokes fields is effectively equivalent to the maximally polarization entangled state

$$
|\Psi\rangle_{\mathrm{AS}, \mathrm{S}} \sim|H\rangle_{\mathrm{AS}}|V\rangle_{\mathrm{S}}+\mathrm{e}^{i\left(\varphi_{1}+\varphi_{2}\right)}|V\rangle_{\mathrm{AS}}|H\rangle_{\mathrm{S}} .
$$

Here, $\varphi_{1(2)}=\Delta \theta_{\mathrm{W}(\mathrm{R})}+\Delta \theta_{\mathrm{AS}(S)}$ represents the phase difference between the two anti-Stokes (Stokes) fields at $\mathrm{PBS}_{1}\left(\mathrm{PBS}_{2}\right)$. As shown in Fig. 2, the phase shift $\Delta \theta_{\mathrm{W}(\mathrm{R})}$ arises from the path difference of the two write (read) beams from $\mathrm{BS}_{2}\left(\mathrm{BS}_{1}\right)$ to the $\mathrm{U}$ and $\mathrm{D}$ ensembles; $\Delta \theta_{\mathrm{AS}(\mathrm{S})}$ arises from the path difference between the two anti-Stokes (Stokes) fields from the $\mathrm{U}$ and $\mathrm{D}$ ensembles to $\mathrm{PBS}_{1}\left(\mathrm{PBS}_{2}\right)$. In the experiment, $\Delta \theta_{\mathrm{W}}+\Delta \theta_{\mathrm{R}}$ and $\Delta \theta_{\mathrm{AS}}+\Delta \theta_{\mathrm{S}}$ are actively stabilized by two Mach-Zehnder interferometers (see the Methods section), respectively. In this way, $\varphi_{1}+\varphi_{2}$ is actively stabilized and fixed to zero.
Table 1 Fidelities of teleporting a photonic qubit at a storage time of $0.5 \mu \mathrm{s}$. For each teleporting state, data was collected for $2 \mathrm{~h}$. The statistical error-that is, \pm 1 standard deviation-is given.

\begin{tabular}{cc}
\hline Original state & Fidelities \\
\hline$|H\rangle$ & $0.865 \pm 0.017$ \\
$|+\rangle$ & $0.737 \pm 0.009$ \\
$|R\rangle$ & $0.750 \pm 0.009$ \\
\hline
\end{tabular}

With an anti-Stokes photon generation probability of 0.003 , the signal-to-noise ratio between the desired $\left(|H\rangle_{\mathrm{AS}}|V\rangle_{\mathrm{S}}\right.$ and $\left.|V\rangle_{\mathrm{AS}}|H\rangle_{\mathrm{S}}\right)$ and unwanted $\left(|H\rangle_{\mathrm{AS}}|H\rangle_{\mathrm{S}}\right.$ and $\left.|V\rangle_{\mathrm{AS}}|V\rangle_{\mathrm{S}}\right)$ components is observed to be $15: 1$, corresponding to a visibility of $87.5 \%$ with a statistical error of $0.4 \%$. This confirms that the $|H\rangle_{A S}|V\rangle_{S}$ and $|V\rangle_{\mathrm{AS}}|H\rangle_{\mathrm{S}}$ terms are two dominant components. Furthermore, to prove the two terms are indeed in a coherent superposition, we also measure the polarization correlation in the $45^{\circ}$ basis. The experimental results exhibit an interference fringe with a visibility of $(82.2 \pm 0.4) \%$, confirming the good quality of atomphoton entanglement.

In the experiment, the initial state to be teleported is prepared using a weak coherent pulse that has the same frequency as an anti-Stokes photon. The probability of containing a single photon for each weak coherent pulse is 0.03 . Without loss of generality, we select horizontal $(|H\rangle), 45^{\circ}(|+\rangle=(1 / \sqrt{2})|H+V\rangle)$ and righthand circular $(|R\rangle=(1 / \sqrt{2})|H+i V\rangle)$ polarizations as our initial states. As shown in Fig. 1, after knowing the BSM results at Alice's site, the atomic excitation at Bob's site is then converted back to a photonic state in a controllable time to analyse the teleportation fidelity.

With emphasis we note that, because the two-photon events from the weak coherent pulses contribute a significant amount of spurious twofold BSM coincidences-which herald nothing but the arrival of two source photons and cannot be distinguished from the true BSM results-a twofold BSM click can only with an average probability of $40 \%$ herald the success of teleportation, given an arbitrary initial state. Therefore, as in previous teleportation experiments $^{9-11}$, in reality our teleportation only occurs posteriorly, that is, conditional on detecting a threefold coincidence. Moreover, owing to the imperfect retrieve, collection and detection efficiency of the teleported states, $30 \%, 75 \%$ and $50 \%$ respectively (see the Methods section), in our experiment the overall teleportation success probability is about $10^{-6}$ per experimental run.

Table 1 shows the experimental result of the teleportation fidelities at a retrieve time of $0.5 \mu$ s. The result shows that the fidelities for different initial states are all well beyond the classical limit of two-thirds, confirming the success of teleportation between photonic and atomic qubits.

To show the ability to store the teleported state in our quantum memory, we further measure the fidelity of teleportation of righthand circular polarization for different retrieve times. The result is shown in Fig. 3. Up to 8 us, the fidelity is still above the classical limit. The fidelity drops down mainly because of the decoherence in the collective atomic state ${ }^{25}$.

In summary, we have demonstrated quantum teleportation between photonic and atomic qubits. The ability to teleport the unknown quantum state of a photonic qubit onto an atomic qubit and then converting it back to a photonic state at a controllable time is essential for the recent quantum-repeater protocols ${ }^{3-5}$ that address the extremely difficult phase stabilization, as required in an original scheme for long-distance quantum communication ${ }^{2}$. However, we note that owing to the low success probability of teleportation and the short lifetime of quantum memory, 


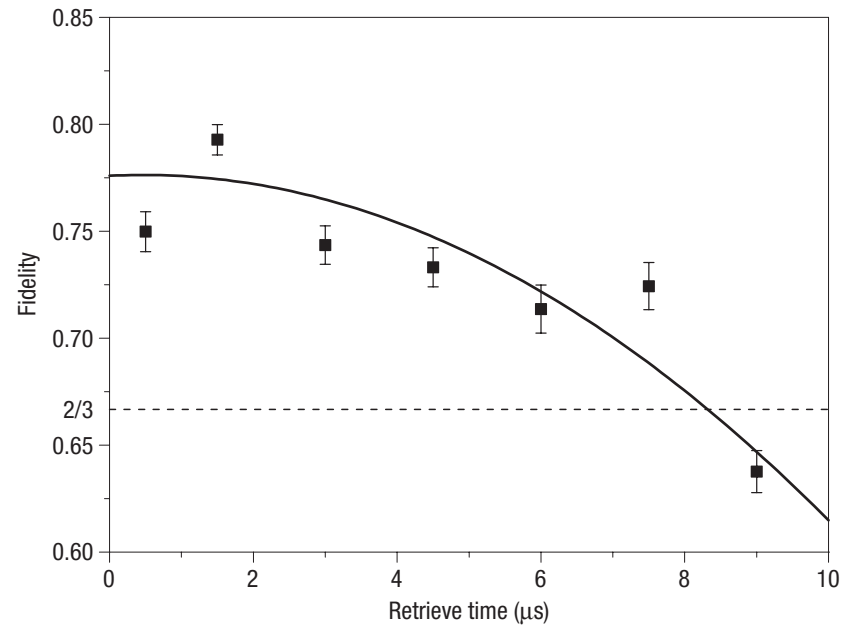

Figure 3 Fidelity of $|R\rangle$ teleportation as a function of storage time. Until $8 \mu$ s, the fidelity is still well beyond the classical limit of $2 / 3$. Each experimental point represents measurements over about $4 \mathrm{~h}$ (on average). The curve is a gaussian fit, owing to the gaussian decay of the retrieve efficiency. The error bars represent the statistical error, that is, \pm 1 standard deviation.

significant improvements are still needed for our method to be useful for practical applications. For example, we could use active feedforward to achieve both a deterministic entanglement source $^{3-5}$ and a high-quality single-photon source ${ }^{25,26}$, by which the overall success of the teleportation rate can be greatly increased while the spurious coincidence is suppressed. This, given our present excitation rate of anti-Stokes photons, would require a lifetime of quantum memory up to $1 \mathrm{~ms}$. Moreover, to achieve long-distance quantum communication, for example, free-space quantum teleportation over $100 \mathrm{~km}$, the same order of storage time is required. To do so, we can confine the atoms in an optical trap and exploiting a clock state to store the collective spin excitation ${ }^{27}$; this could potentially extend the lifetime up to $1 \mathrm{~s}$. Finally, comparing former photonic teleportation ${ }^{8-10}$, where the coherence time of down-converted photons is only about a few hundred femtoseconds, the narrowband feature of our anti-Stokes photon source (coherence time $\sim 25 \mathrm{~ns}$ ) makes the overlap of independent photon wave packets from distant sites much easier. This advantage together with the feasible long-lifetime quantum memory may provide an ideal solution for large-scale communications, for example, satellite-based quantum communication ${ }^{28,29}$.

\section{METHODS}

\section{EXPERIMENTAL CYCLES}

In the experiment, the MOT is loaded for $20 \mathrm{~ms}$ at a repetition rate of $40 \mathrm{~Hz}$. The magnetic field and cooling beams are then quickly switched off, whereas the repumping beams stay on for $0.5 \mathrm{~ms}$ before being switched off to prepare the atoms in the initial $F=2$ ground state $|a\rangle$. Then, within another $4.5 \mathrm{~ms}$, experimental trials (each consisting of successive write, read and repumping pulses) are repeated with a controllable period depending on the desired retrieve time of the teleported state.

\section{PHASE LOCKING}

To stabilize the phase $\varphi_{1}+\varphi_{2}$ in equation (3) actively, two Mach-Zehnder interferometers are used as shown in Fig. 2. Because the spatial mode of the anti-Stokes (Stokes) field and the write (read) beam have an angle of $3^{\circ}$, we cannot lock the phase $\varphi_{1}\left(=\Delta \theta_{\mathrm{W}}+\Delta \theta_{\mathrm{AS}}\right)$ and $\varphi_{2}\left(=\Delta \theta_{\mathrm{S}}+\Delta \theta_{\mathrm{R}}\right)$ directly. However, we can lock the phase of $\Delta \theta_{\mathrm{W}}+\Delta \theta_{\mathrm{R}}$ and $\Delta \theta_{\mathrm{AS}}+\Delta \theta_{\mathrm{S}}$ separately. To stabilize the phase of $\Delta \theta_{\mathrm{W}}+\Delta \theta_{\mathrm{R}}$, the read beam is switched on during the
$20 \mathrm{~ms}$ MOT loading stage, and is used as the locking beam (Fig. 2a). During the $5 \mathrm{~ms}$ experimental stage, the shutter is switched off. The interference signal can be used as the error signal of a standard proportional-integrate locking circuit. The error signal is normalized by the duty cycle and then sent to the home-built proportional-integrate circuit. By controlling the voltage of the piezo-electric device (P1), we can lock the phase $\Delta \theta_{\mathrm{W}}+\Delta \theta_{\mathrm{R}}$ to a set value. To stabilize the phase $\Delta \theta_{\mathrm{AS}}+\Delta \theta_{\mathrm{S}}$, a further locking beam polarized at $45^{\circ}$ with the frequency of the read beam is sent in at the angle of the first-order diffraction of the acousto-optic modulator (AOM; Fig. $2 \mathrm{~b}$ ) during the MOT loading stage. Going through the AOM, the locking beam is overlapped with the Stokes and anti-Stokes beams. As the anti-Stokes and Stokes light are perpendicularly polarized, the output of the locking beam is from another port of $\mathrm{PBS}_{1}$. After the locking beam goes through a polarizer at $45^{\circ}$, the interference signal can be detected by a photodiode and used to lock the phase $\Delta \theta_{\mathrm{AS}}+\Delta \theta_{\mathrm{S}}$. During the experimental stage, the shutter and the RF power of the AOM are all switched off to prevent the leakage of the locking beam from entering into the anti-Stokes-Stokes channels. In this way, the overall phase of $\varphi_{1}+\varphi_{2}$ is actively locked.

\section{NOISE ESTIMATION}

In our experiment, the intensity of the write pulses is adjusted such that in each experimental run the probability of creating an anti-Stokes photon behind $\mathrm{PBS}_{1}$ is $p_{\mathrm{AS}} \sim 0.003$. The intensity of the read pulses is about 70 times higher than the write pulses. Under this condition, we achieve a retrieve efficiency of $\gamma \sim 30 \%$. After each write and read process, the probability of emitting a single photon in Stokes mode (denoted by $p_{\mathrm{S}}$ ) is measured to be $\sim 0.004$. In each weak coherent pulse, the probability of containing a single photon is $p_{0} \sim 0.03$.

Thus, our threefold coincidence would mainly have three components: (1) Coincidence among a single photon of the initial state from the weak coherent beam, an anti-Stokes photon and a successfully retrieved Stokes photon, which is the desired event and has a probability of $\sim(1 / 2) p_{\mathrm{AS}} p_{0} \gamma \eta^{3}$. Here, $\eta$ is the average overall detection efficiency of our single-photon detectors, that is, the product of the collection efficiency $(\sim 75 \%)$ and the detection efficiency of the detectors $(\sim 50 \%)$. (2) Spurious coincidence contributed by a two-photon event from the weak coherent pulse and a single-photon event in Stokes mode. In teleportation of $|+\rangle$ and $|R\rangle$ states, the probability of registering such threefold coincidence is given by $(1 / 4) p_{0}^{2} p_{\mathrm{S}} \eta^{3}$. However, because only $\left|\Psi^{ \pm}\right\rangle$is analysed in our BSM, in teleportation of the $|H\rangle$ state, the two-photon event from the weak coherent beam will not lead to a two-photon coincidence in our BSM and thus also not to a spurious threefold coincidence. (3) Spurious coincidence contributed by double emission from the atomic ensembles and one retrieved Stokes photon, which has a probability of $(1 / 4) p_{A S}^{2}\left(2 \gamma \eta-(\gamma \eta)^{2}\right) \eta^{2}$.

Moreover, the probability of dark counts in each detector is about $10^{-5}$ per trial, implying a signal-to-noise ratio of better than 100:1. And the errors in polarization are less than $1 \%$. These two errors are thus negligible. Denote the probability of the desired threefold coincidence as $S=(1 / 2) p_{\mathrm{AS}} p_{0} \gamma \eta^{3}$ and the probability of the spurious one as $N=(1 / 4) p_{0}^{2} p_{\mathrm{S}} \eta^{3} \kappa_{\phi}+(1 / 4) p_{\mathrm{AS}}^{2}\left(2 \gamma \eta-(\gamma \eta)^{2}\right) \eta^{2}\left(\phi\right.$ is the initial state, $\kappa_{\mathrm{H}}=0$ and $\left.\kappa_{+}=\kappa_{\mathrm{R}}=1\right)$. Taking into account the imperfection of the entanglement source, we can thus estimate the final fidelity for $|H\rangle$ teleportation by $f=(S(1+V) /(2+N / 2)) /(S+N)$, where $V \approx 0.88$ is the entanglement visibility in the $H / V$ basis. A simple calculation shows that the final fidelity should be around 0.90 , which is in good agreement with our experimental fidelity $0.865 \pm 0.017$. The slight difference is probably due to the neglected dark count and polarization errors.

In teleportation of $|+\rangle$ and $|R\rangle$ states, the experimental fidelities are much lower. This is because, on the one hand we have one more spurious threefold coincidence contribution, that is, $(1 / 4) p_{0}^{2} p_{S} \eta^{3}$. More importantly, the imperfect overlap of the wave packets on the beam splitter, typically around $90 \%$ in our experiment $\mathrm{t}^{23}$, will further reduce the fidelities significantly. However, note that such imperfection has no effect on the $|H\rangle$ teleportation fidelity. Taking these into account, a similar calculation shows that the final fidelity for $|+\rangle$ and $|R\rangle$ teleportation should be around 0.79 . Given the neglected dark count and polarization errors, our experimental results again agree well with the calculated fidelities.

\section{ASYMMETRIC SYSTEMATIC ERROR}

Experimentally, the teleportation fidelities of two orthogonal input states under a complete basis could be significantly different owing to various asymmetric systematic errors, for example, caused by asymmetric atom-photon entanglement, state preparation or state analysis. In our experiment, before 
data collection, various efforts have been made to minimize such asymmetric error. First, the initial polarization state to be teleported is prepared with an extinction ratio of better than 500:1. Second, in entanglement preparation and BSM, high-quality PBSs are selected and adjusted to achieve an extinction ratio of better than 300:1 for both $H$ and $V$ polarization. Third, the excitation probability of each ensemble is adjusted properly so that we have a symmetric entangled state (the asymmetric property of the entangled state is verified to be less than $1 \%)$. Finally, in polarization analysis of the teleported states, an extinction ratio of better than 100:1 is achieved by using a high-quality polarizer. In addition, we have investigated the teleportation fidelities of two states with opposite polarizations in independent experiments and find that the overall asymmetric systematic error of our method is less than $1 \%$.

Received 9 May 2007; accepted 6 December 2007; published 20 January 2008.

\section{References}

1. Bennett, C. H. et al. Teleporting an unknown quantum state via dual classical and Einstein-Podolsky-Rosen channels. Phys. Rev. Lett. 70, 1895-1899 (1993).

2. Duan, L.-M., Lukin, M. D., Cirac, J. I. \& Zoller, P. Long-distance quantum communication with atomic ensembles and linear optics. Nature 414, 413-418 (2001).

3. Zhao, B., Chen, Z.-B., Chen, Y.-A., Schmiedmayer, J. \& Pan, J.-W. Robust creation of entanglement between remote memory qubits. Phys. Rev. Lett. 98, 240502 (2007).

4. Jiang, L., Taylor, J. M. \& Lukin, M. D. Fast and robust approach to long-distance quantum communication with atomic ensembles. Phys. Rev. A 76, 012301 (2007).

5. Chen, Z.-B., Zhao, B., Chen, Y.-A., Schmiedmayer, J. \& Pan, J.-W. Fault-tolerant quantum repeater with atomic ensembles and linear optics. Phys. Rev. A 76, 022329 (2007).

6. Briegel, H.J., Dür, W., Cirac, J. I. \& Zoller, P. Quantum repeaters: The role of imperfect local operations in quantum communication. Phys. Rev. Lett. 81, 5932-5935 (1998).

7. Gottesman, D. \& Chuang, I. L. Demonstrating the viability of universal quantum computation using teleportation and single-qubit operations. Nature 402, 390-393 (1999).

8. Knill, E., Laflamme, R. \& Milburn, G. J. A scheme for efficient quantum computation with linear optics. Nature 409, 46-52 (2001).

9. Bouwmeester, D. et al. Experimental quantum teleportation. Nature 390, 575-579 (1997).

10. Pan, J.-W., Bouwmeester, D., Weinfurter, H. \& Zeilinger, A. Experimental entanglement swapping: Entangling photons that never interacted. Phys. Rev. Lett. 80, 3891-3894 (1998).

11. Zhao, Z. et al. Experimental demonstration of five-photon entanglement and open-destination teleportation. Nature 430, 54-58 (2004).
12. Matsukevich, D. N. \& Kuzmich, A. Quantum state transfer between matter and light. Science 306, 663-666 (2004).

13. Eisaman, M. D. et al. Electromagnetically induced transparency with tunable single-photon pulses. Nature 438, 837-841 (2005).

14. Chanelière, T. et al. Storage and retrieval of single photons transmitted between remote quantum memories. Nature 438, 833-836 (2005).

15. Riebe, M. et al. Deterministic quantum teleportation with atoms. Nature 429, 734-737 (2004).

16. Barrett, M. D. et al. Deterministic quantum teleportation of atomic qubits. Nature $\mathbf{4 2 9}$, 737-739 (2004).

17. Furusawa, A. et al. Unconditional quantum teleportation. Science 282, 706-709 (1998).

18. Sherson, J. F. et al. Quantum teleportation between light and matter. Nature 443, 557-560 (2006).

19. Hammerer, K., Polzik, E. S. \& Cirac, J. I. Teleportation and spin squeezing utilizing multimode entanglement of light with atoms. Phys. Rev. A 72, 052313 (2005).

20. Kozhekin, A. E., Mølmer, K. \& Polzik, E. S. Quantum memory for light. Phys. Rev. A 62, 033809 (2000).

21. Schmitt-Manderbach, T. et al. Experimental demonstration of free-space decoy-state quantum key distribution over $144 \mathrm{~km}$. Phys. Rev. Lett. 98, 010504 (2007).

22. Peng, C.-Z. et al. Experimental long-distance decoy-state quantum key distribution based on polarization encoding. Phys. Rev. Lett. 98, 010505 (2007).

23. Yuan, Z.-S. et al. Synchronized independent narrow-band single photons and efficient generation of photonic entanglement. Phys. Rev. Lett. 98, 180503 (2007).

24. Mattle, K., Weinfurter, H., Kwiat, P. G. \& Zeilinger, A. Dense coding in experimental quantum communication. Phys. Rev. Lett. 76, 4656-4659 (1996).

25. Chen, S. et al. Deterministic and storable single-photon source based on a quantum memory. Phys. Rev. Lett. 97, 173004 (2006).

26. Matsukevich, D. N. et al. Deterministic single photons via conditional quantum evolution. Phys. Rev. Lett. 97, 013601 (2006).

27. Harber, D. M., Lewandowski, H. J., McGuirk, J. M. \& Cornell, E. A. Effect of cold collisions on spin coherence and resonance shifts in a magnetically trapped ultracold gas. Phys. Rev. A 66, 053616 (2002).

28. Aspelmeyer, M. et al. Long-distance free-space distribution of quantum entanglement. Science 301, 621-623 (2003).

29. Peng, C.-Z. et al. Experimental free-space distribution of entangled photon pairs over a noisy ground atmosphere of $13 \mathrm{~km}$. Phys. Rev. Lett. 94, 150501 (2005).

\section{Acknowledgements}

This work was supported by the Deutsche Forschungsgemeinschaft (DFG), the Alexander von Humboldt Foundation, the Chinese Academy of Sciences (CAS), the National Fundamental Research Program (Grant No. 2006CB921900) and NNSFC.

Correspondence and requests for materials should be addressed to Y.-A.C. or J.-W.P.

Reprints and permission information is available online at http://npg.nature.com/reprintsandpermissions/ 